\title{
Placental site trophoblastic tumor presented with unusual presentation
}

\section{Rajdeep Meena, Santosh Meena*}

Department of Obstetrics and Gynecology, Government Medical College, Kota-324001, Rajasthan, India

Received: 30 June 2016

Accepted: 05 August 2016

\section{*Correspondence:}

Dr. Santosh Meena,

E-mail: drmithlesh.meena2608@gmail.com

Copyright: () the author(s), publisher and licensee Medip Academy. This is an open-access article distributed under the terms of the Creative Commons Attribution Non-Commercial License, which permits unrestricted non-commercial use, distribution, and reproduction in any medium, provided the original work is properly cited.

\begin{abstract}
Placental site trophoblastic tumor (PSTT) is a rarest type of gestational trophoblastic tumor. It is rising from the abnormal proliferation of intermediate trophoblastic cells with occasional multinuclear giant cells, with the potential for local invasion and metastasis. A 28 year old G4P3 woman presented with 4 months Amenorrhea, large uterine mass and plateau low level of serum human chorionic gonadotropin (hCG) which raised the possibility of PSTT. Since whole myometrium has been substituted with the large tumoral mass, hysterectomy was considered despite the young age of the patient. The patient has been in remission 12 months postoperatively without receiving any adjuvant treatment. It seems that plateau low levels of hCG in the presence of amenorrhea should prompt the possibility of PSTT.
\end{abstract}

Keywords: Placental site trophoblastic tumor, Gestational trophoblastic neoplasia, Metastasis

\section{INTRODUCTION}

PSST was first described as an exaggerated expression of the invasion nature of normal trophoblastic tissue and named as trophoblastic pseudotumor in $1976 .{ }^{1}$ Placental site trophoblastic tumors, the rarest type in this group, originates from implantation-site intermediate trophoblastic cells which infiltrate both myometrium and blood vessels. $70 \%$ patients' localized to the uterus, while $30 \%$ of patients had metastasis Lesions at first presentation. As a result, the symptoms of PSTT are variable, including amenorrhea, irregular bleeding, abdominal pain, uterine rupture and vaginal metastasis. ${ }^{3}$ This tumor is fairly resistant to chemotherapy and hysterectomy is valuable as primary treatment.

\section{CASE REPORT}

A 28 year old G4P3 woman presented with 4 months amenorrhea with vaginal growth. Her last pregnancy was delivered by spontaneous vaginal delivery by spontaneous vaginal delivery 3 year previously.
History of dilatation and evacuation for molar pregnancy done in last 6 months. On examination, she had significant pallor with stable vital parameters. Her hemoglobin was $6 \mathrm{gm} \%$. Her abdominal examination revealed 16 weeks smooth firm mass with freely mobile, which seemed to be arising from the pelvis. Her pelvic examination confirmed the mass to be mobile with uterus and was of firm consistency. The patient had active trickling of blood per vaginum. The ultrasound findings revealed that uterus is bulky of approximately $15 \times 9 \times 7$ $\mathrm{cm}$, endometrium is normal; fluid with internal echoes and multiple cystic lesions of varying sizes seen in endometrial cavity and uterine myometrium may be molar degeneration. $\beta$ HCG - 292 $\mu \mathrm{IU} / \mathrm{ml}$. The patient had a normal chest X-ray and ECG.

The patient was advised admission. During the hospital stay, patient was transfused with 4 units of packed red blood cells to be rise hemoglobin. After informed consent, laparotomy was planned, intraoperative finding there were presence of multiple nodules on anterior and posterior wall of uterus, bilateral adnexa normal when 
uterus exteriout tooriential bleeding occurred hysterectomy done. Specimen revealed a bulky uterus of approximately $14-16 \mathrm{~cm}$. On cut section, the large amount of clotted blood was evident within the uterus. Upon removal of blood clots, approximately $6 \times 8 \mathrm{~cm}$ mass was felt on posterior aspect of uterine wall. Along with uterus and cervical tissue, fluids from paracolic gutter and pouch of Douglas. Postoperative period was eventful. Histopathology report disclosed that it was endometrium - placental site trophoblastic tumor, myometrium show sheets of intermediate trophoblast separating the muscle fibres. Postoperatively $\beta$ hCG was followed up serially. During follow up period, the patient was evaluated regularly with hemoglobin, ultrasound, chest X-ray, $\beta$ hCG.

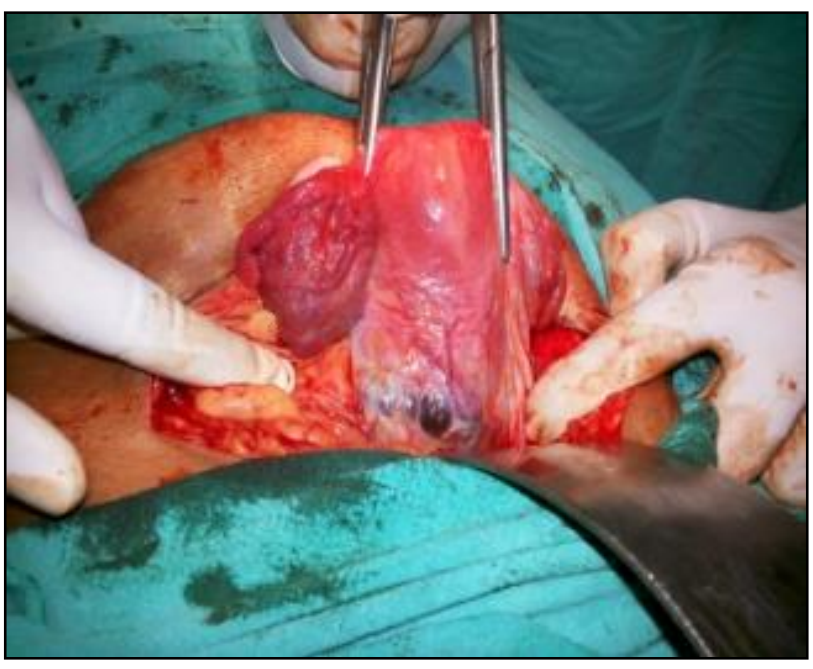

Figure 1: Anterior wall of uterus.

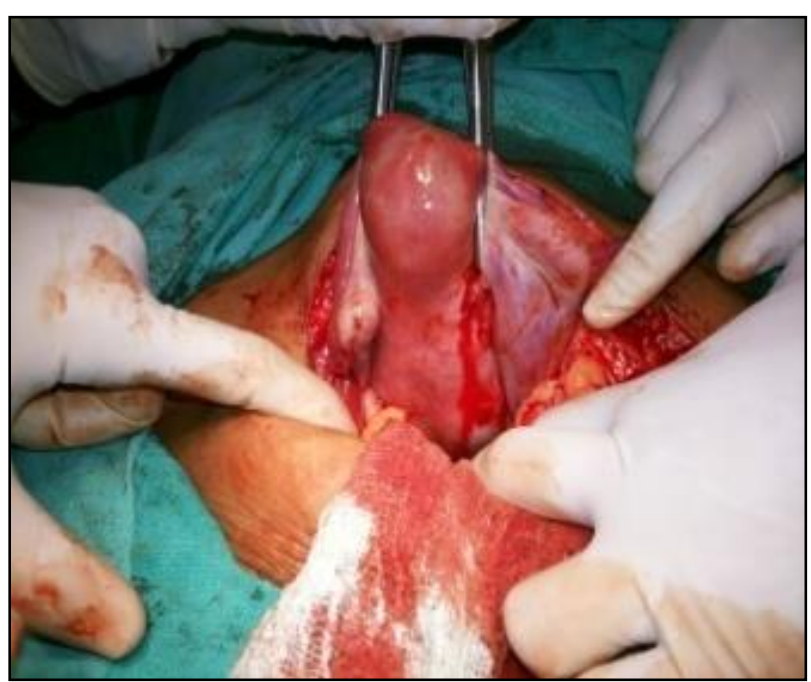

Figure 2: posterior wall of uterus.

\section{DISCUSSION}

Gestational trophoblastic disease (GTD) defines a heterogeneous group of interrelated lesions arising abnormal proliferation of trophoblastic epithelium of placenta. All GTDS are characterized by the distinct tumor marker human chorionic gonadotropin. ${ }^{2}$ Subtype of GTD include partial and complete hydatiform mole and gestational neoplasia (GTN). GTNs including invasive mole, choriocarcinoma, and placental site trophoblastic tumor (PSTT) have the potential for local invasion and metastasis. PSST is a tumor of intermediate trophoblast, and accounts for $1-2 \%$ gestational trophoblastic neoplasm. ${ }^{1}$ Sometime, it is difficult to distinguish PSST from other form of GTN or non-trophoblastic tumors just as the referring hospital on base of morphological and clinical data.

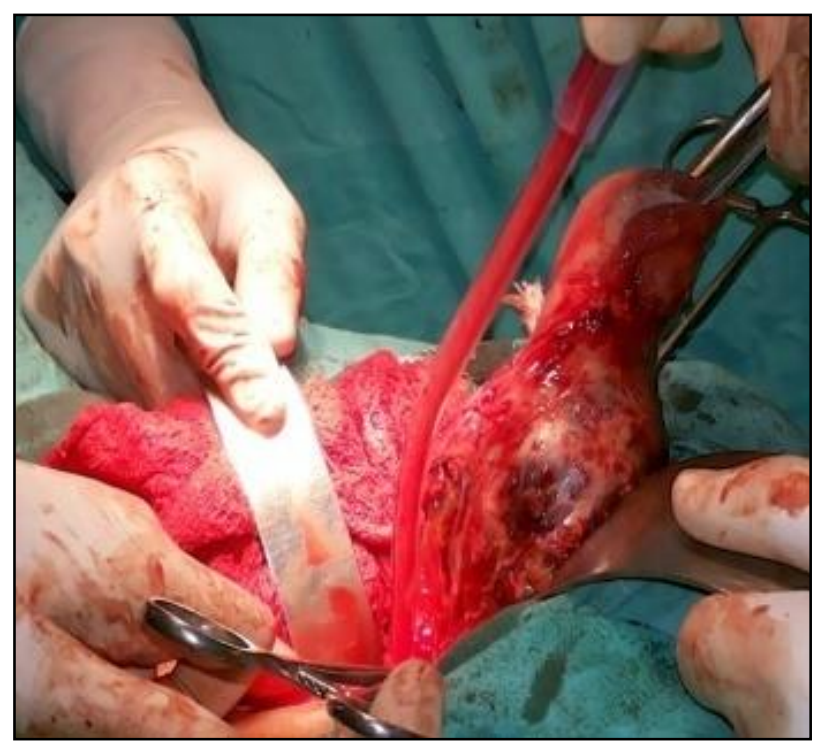

Figure 3: Placental site trophoblastic tumor.

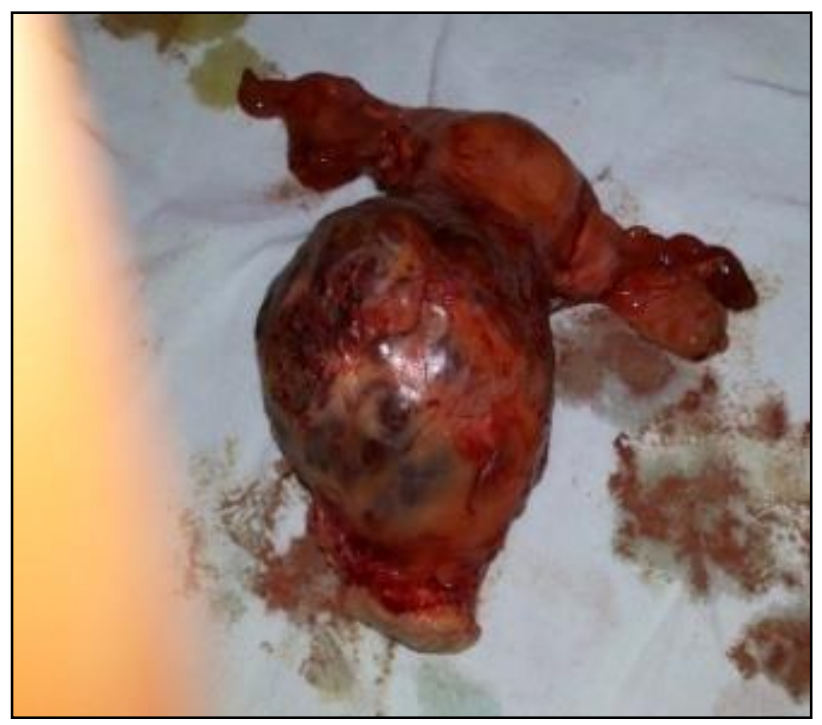

Figure 4: GTNs including invasive mole, choriocarcinoma.

The symptoms of gestational trophoblastic disease usually occur after delivery or abortion. According to the epidemiological retrospective survey, gestational trophoblastic neoplasia, $60 \%$ was secondary to 
hydatiform mole, $30 \%$ to abortion, and $10 \%$ secondary to full term pregnancy or ectopic pregnancy. However, PSST may complicate or follow any type of normal or abnormal gestations (hydatiform mole) and its interval may vary from weeks to years after the preceding pregnancy. Comparing with the high level in other type of GTN, the serum-hCG in PSST may be mild elevated or just at normal level, which was no help for diagnosis preoperatively. Over $30 \%$ of patients have metastatic lesions at first presentation, such as the peritoneum, lung, liver, brain. The symptoms of PSST are variable, including amenorrhea, irregular bleeding pain, uterine rupture.

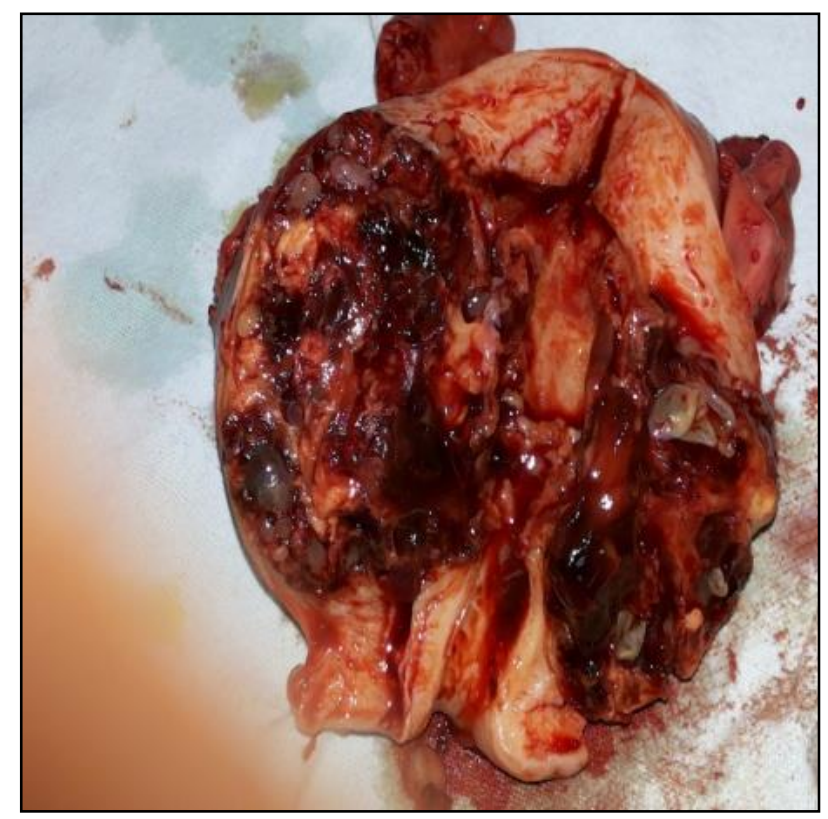

Figure 5: Trophoblastic tumor.

However, identification of the GTN subtype and differentiating them from other tumors is important clinically because the therapeutic approaches towards this disease are difference .In contrast to other forms of GTDs, because of its low chemo sensitivity, surgery is main treatment option. ${ }^{4}$

\section{CONCLUSION}

PSST is a well-recognized but rare form of GTD with variable presentations. Most of tumors are localized in the uterus. Due to its rarity and variable manifestation it presents a diagnostic challenge and treatment dilemma. The aim of presenting this case of uterine PSST was to illustrate the difficulties in the diagnosis of this tumor and how to delay in the appropriate management. Hysterectomy seems to be the appropriate treatment of a patient with confined tumor in the uterus, but metastatic patients also need chemotherapy.

\section{Funding: No funding sources \\ Conflict of interest: None declared \\ Ethical approval: Not required}

\section{REFERENCES}

1. Kurman RJ, Scully RE, Nrris HJ. Trophoblastic pseudotumor of the uterus. An exaggerated form of syncytial endometritis, simulating a malignant tumor. Cancer. 1976;7:1214-26.

2. Soper JT, Mutch DG, Schink JC American College of Obstetrics and Gynecologists. Diagnosis and treatment of gestational trophoblastic disease.ACOG practice Bulletin: No. 53. Gynecol Oncol. 2004;93(3):575-85.

3. Hassadia A, Gillespie A, Tidy J, Everard RGNJ, Wells M, Coleman R, et al. Placental site trophoblastic tumor: Clinical features and management. Gynecol Oncol. 2005;99(3):603-7.

4. Sudha CP, Sahana M. Chemoresistant gestational trophoblastic neoplasia: a case report. J Clin Diagn Res. 2014;8:OD12-3.

Cite this article as: Meena R, Meena S. Placental site trophoblastic tumor presented with unusual presentation. Int J Reprod Contracept Obstet Gynecol 2016;5:3218-20. 